\title{
铝合金熔体在多孔介质中的渗流过程及多孔 铝合金
}

何德坪 ${ }^{*}$, 何思渊，顾菲菲，黄可

东南大学, 南京 210096

* 通讯作者, E-mail: dphe@seu.edu.cn

收稿日期：2008-11-17; 接受日期：2008-12-17

摘要以高技术需求的多孔铝合金为研究对象, 揭示了铝合金熔体在多孔介质中 的渗流过程及影响规律; 根据相似原理采用渗流模拟法揭示了模拟液在多孔介质中 渗流的规律, 与熔体渗流过程结果吻合良好; 建立了熔体在多孔介质中渗流, 控制 多孔铝合金孔结构的物理模型并与实验吻合, 以控制孔结构; 在上述单一孔径多孔 铝合金基础上, 发展了梯度孔径及周期调制的新型多孔铝合金, 获得了更佳的声学 性能.

\section{关键词}

多孔铝合金

熔体渗流

模拟渗流

孔结构

孔径调制

\section{1 引言}

自H A Kucheck ${ }^{[1]}$ 探索多孔铝合金以来, 这一领 域开始发展. 以毫米级孔径 $d$ 及较高孔隙率 $P$ 为结构 特征的通孔多孔铝合金，与用作过滤的微米级通孔 粉末冶金多孔金属不同，具有轻质 $(\rho<1)$, 高比强，高 比刚度, 及与流通特性相关的消声，吸声，散热和阻 尼减振, 能量吸收, 电磁屏蔽等多功能兼容性能. 近 5 年由于高技术成功应用，铝合金熔体在毫米级多孔 介质中渗流过程和通孔多孔铝合金(porous Al alloy) 引起高度重视, 成为超轻多孔金属中迅速发展的科 学前沿领域.

本文系统概括了铝合金熔体在多孔介质中渗流 过程及多孔铝合金如下新进展:

(1) 铝合金熔体在可去除多孔介质中渗流、凝固 形成通孔多孔铝合金(考虑热交换) $)^{[2]}$.

（2）采用物理模拟方法，揭示铝合金熔体在多孔 介质间渗流规律 $(\text { 不考虑热交换 })^{[3]}$.
(3) 通孔多孔铝合金孔结构控制(附加孔隙率)的 物理模型 $[4]$.

(4) 在上述单一孔径的基础上发展了梯度孔径 及周期调制多孔铝合金 ${ }^{[5]}$.

上述以多学科视角系统概括这一前沿领域的新 进展尚未见到报道.

\section{2 铝合金熔体在多孔介质间渗流、凝固形 成多孔铝合金}

\section{1 通孔多孔铝合金渗流制备原理及多样化孔 结构}

(i) 铝合金熔体渗流形成多孔铝合金的基本原理. 在压力驱动下，铝合金熔体在可除去多孔介质间渗流 和凝固，形成多孔介质和铝合金复合体，去除多孔介 质形成通孔多孔铝合金. 控制多孔介质的直径及堆积 密度以控制多孔铝合金的孔径 $d$ 及孔隙率 $P$. 显然，渗 流过程铝熔体与多孔介质存在热交换及润湿现象. 
表 1 通孔多孔铝合金的制备方法和孔结构

\begin{tabular}{|c|c|c|c|c|c|c|}
\hline 序号 & 制备方法 & 颗粒及预制体 & 孔隙率 $/ \%$ & 孔径 $/ \mathrm{mm}$ & 孔隙率梯度 $/ \% \cdot \mathrm{dm}^{-1}$ & 文献 \\
\hline (1) & 熔体渗流法 & 可溶性 $\mathrm{NaCl}$ 颗粒 & $57.3 \sim 70.0$ & $0.5 \sim 7.0$ & $0 \sim 3.0$ & {$[2]$} \\
\hline (2) & 高压渗流法 & 可燃低熔点预制体 & $70.0 \sim 85.0$ & $2.0 \sim 3.0$ & $\approx 0$ & [6] \\
\hline (3) & 塑料颗粒压缩变形法 & HDPE、LDPE、PP、TPV & $74.5 \sim 94.0$ & $2.0 \sim 6.0$ & $0 \sim 10.0$ & {$[7]$} \\
\hline (4) & 熔模铸造法 & 石膏、聚氨酯海绵 & $92.0 \sim 96.0$ & $2.5 \sim 5.5$ & $\approx 0$ & {$[8]$} \\
\hline
\end{tabular}

要保证熔体不完全包围多孔介质，形成能去除多孔介 质的通孔度，这是形成通孔多孔铝合金的必要条件。

（ii）多样化孔结构. 采用多种方法宽范围调节通 孔多孔铝合金的孔结构: 孔径，孔隙率，形成多样化 的多孔铝合金，以满足不同高技术需求. 表 1 列出部 分多孔铝合金的制备方法及孔结构.

根据高技术需求对通孔泡沫铝孔隙率范围的需 求，通过对比研究，采取序号(1)的方法制备高比强通 孔多孔铝合金.

\section{2 考虑热交换时，铝合金熔体在多孔介质间渗 流过程的物理模型}

简要物理模型如下: 具有过热度的铝合金熔体在 凝固潜热消失以前的渗流长度 $L$, 包括 $L_{1}$ 及 $L_{2}, L$ 很短 因而可以将其线性化处理; 过热温度为 $T_{\mathrm{L}}$ 的铝合金 熔体在压力驱动下渗入低于 $T_{\mathrm{L}}$ 的预热温度 $T_{\mathrm{P}}$ 颗粒之 间区域 I，其长度为 $L_{1}$ ，该区域包括铝合金熔体和颗 粒。在渗流过程中，铝合金熔体 $T_{\mathrm{L}}$ 与颗粒预热温度 $T_{\mathrm{P}}$ 的颗粒进行热交换，从而降低铝合金熔体的温度; 进 入熔体过热度为 0 , 但存在凝固潜热释放的区域 II, 该区域包括铝合金熔体、颗粒及凝固的铝合金 $\mathrm{SM}$, 其长度为 $L_{2}$, 在 II 区内渗流直至前沿形成固相铝合金 SM而停止，见图 1 .

制备高比强通孔多孔铝合金的渗流装置原理图 见图 2 .

从铝合金熔体在颗粒间渗流的传热及渗流的物 理模型可以获得如下表示渗流能力及长度的数学表 达式 ${ }^{[9,10]}$ :

$$
L=\frac{K\left(T_{\mathrm{L}}-T_{\mathrm{M}}\right)}{(1-P) \rho_{\mathrm{p}} C_{\mathrm{p}}\left(T_{\mathrm{M}}-T_{\mathrm{P}}\right) u},
$$

该式可简化为 $L=f\left(T_{\mathrm{L}}, T_{\mathrm{P}}\right)$. 式中 $L$ 为铝合金熔体的 渗流长度, $T_{\mathrm{L}}$ 为铝合金熔体的过热温度; $T_{\mathrm{M}}$ 为铝合金 熔体的凝固温度; $T_{\mathrm{P}}$ 为颗粒的预热温度; $K$ 为铝合金

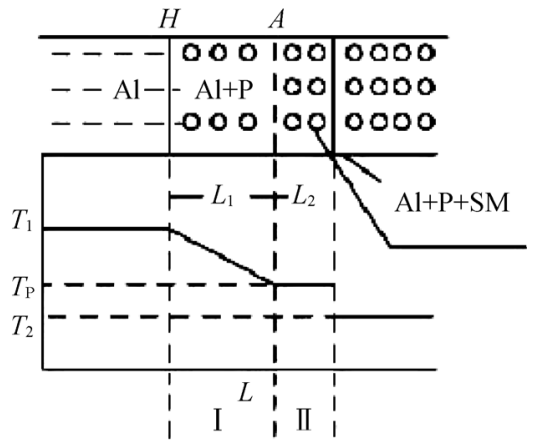

图 1 铝熔体渗流模型与温度分布

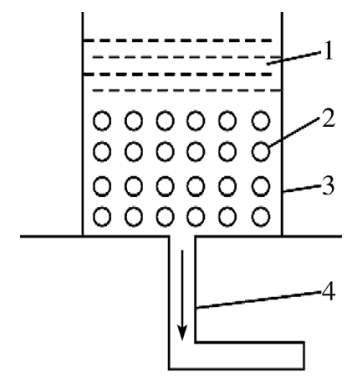

图 2 渗流法原理图

1. 铝熔体; 2. 填料颗粒; 3. 模具; 4. 接真空系统

熔体的热传导率; $C_{\mathrm{P}}$ 为颗粒的比热; $\rho_{\mathrm{P}}$ 为颗粒的密度; 为颗粒孔隙率; $u$ 为铝液移动速度.

由(1)式可见，铝合金熔体渗流长度与(1)铝合金 熔体温度 $T_{\mathrm{L}}$, (2)颗粒预热温度 $T_{\mathrm{P}}$ 有密切关系, 提高(1) 及(2)可显著提高铝合金熔体在多孔介质间的渗流能 力. 研究表明 [9] 铝熔体在多孔介质间渗流的方向不 同(上吸，下吸)渗流规律基本相同。

\section{3 多孔铝合金制备的影响因素}

在相同条件下，研究了铝合金熔体的预热温度 $T_{\mathrm{L}}$ 、颗粒的预热温度 $T_{\mathrm{P}}$ 、颗粒的直径 $d$ 等因素对渗流 长度 $L$ 的影响, 部分试验结果见表 2. 
表 2 试验部分结果

\begin{tabular}{cccccc}
\hline 试验编号 & 铝液温度 $T_{\mathrm{L}} /{ }^{\circ} \mathrm{C}$ & 颗粒预热温度 $T_{\mathrm{p}}{ }^{\circ} \mathrm{C}$ & 外加压力 $p / \mathrm{MPa}$ & 颗粒尺寸 $d / \mathrm{mm}$ & 渗流长度 $L / \mathrm{mm}$ \\
\hline 1 & 760 & 20 & 0.01 & 2.5 & 3.5 \\
2 & 760 & 200 & 0.02 & 4.5 & 13.83 \\
3 & 760 & 260 & 0.03 & 5.5 & 115.3 \\
4 & 760 & 300 & 0.04 & 4.5 & 134.2 \\
5 & 740 & 260 & 0.01 & 5.5 & 106.3 \\
6 & 720 & 200 & 0.03 & 106.3 \\
\hline
\end{tabular}

从表 2 可见, 提高熔体预热温度 $T_{L}$ 和颗粒预热温 度 $T_{P}$, 可以提高熔体渗流能力, 这与上述物理模型结 果相吻合.

综上所述，采用熔体渗流法制备通孔多孔铝合 金，通过提高铝熔体预热温度、颗粒预热温度及合适 的渗流压力，可以获得大长度通孔多孔铝合金.

\section{3 铝合金熔体在毫米级多孔介质中渗流过 程的物理模拟}

铝合金熔体在多孔介质中渗流过程是制备多孔 铝合金及微米级颗粒高强度复合材料的关键，这一 领域最初从数学方法研究微米级颗粒的渗流过 程 ${ }^{[11,12]}$. 前者与后者的差异为: 前者采用毫米级的可 去除颗粒以形成多孔铝合金，而后者则将微米级颗 粒作为强化相保存于复合材料中.

本文在渗流模拟的基础上，获得铝合金熔体在 毫米级多孔介质间渗流的一系列规律，从而为渗流 法制备多孔铝合金奠定了必要的基础 ${ }^{[13]}$.

\section{1 铝合金熔体在多孔介质中渗流模拟的原理及 方法}

模拟渗流试验：采用合适的常温透明模拟液，及 有机透明的渗流模拟装置 (模型 $\mathrm{M}$ ) 及同样尺寸 $\Phi 70 \times 150$ 的熔体渗流原型装置(原型 $\mathrm{P}$ ) ; 多孔介质颗 粒(球形, $d=1.5 \mathrm{~mm}$, 颗粒堆积孔隙率为 $32 \%$ ), 模拟 渗流采用高精度压力传感器(外径 $\Phi 3 \mathrm{~mm}$ ), 5 个传感 器沿轴向间距为 $75 \mathrm{~mm}$ 均匀分布, 见图 3.

假设: (1)由试验获得的渗流时间小于 $3 \mathrm{~s}$, 为高速 渗流. 文献[11]研究表明只要速度大于 $1 \mathrm{~mm} / \mathrm{s}$, 可以 忽略熔体与多孔介质间的热交换, 可将渗流过程的 熔体温度及黏度视为常数. (2)由于毫米级的多孔介 质及较高孔隙率, 铝熔体及模拟液与多孔介质表面

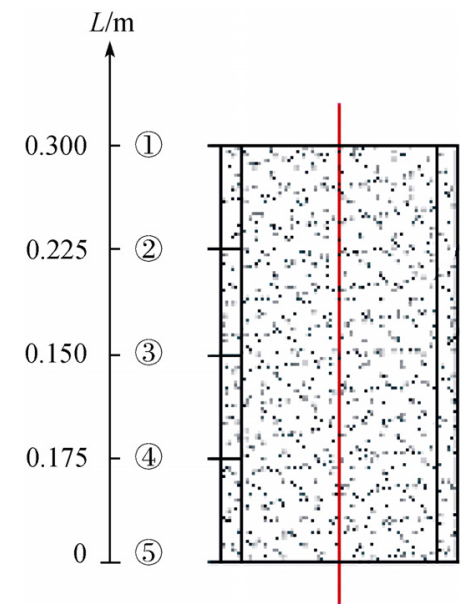

图 3 模拟渗流系统压力传感器的坐标示意图

性能差引起的附加压力差与较高的渗流压力相比甚 小, 可忽略不计. 原型 $(\mathrm{P})$ 及模型 $(\mathrm{M})$ 渗流试验结果表 明上述假设是正确的.

渗流模拟相似原则: 根据流体流动相似原理，要 求原型与模型的流动几何相似、运动相似和动力相似, 以及初始条件和边界条件相似 ${ }^{[14]}$. 原型中液态铝的 渗流是非恒定渗流，所受作用力有重力、黏滞力和压 力. 只有当弗劳德准则、雷诺准则同时满足才能保证 模型和原型的渗流运动相似 ${ }^{[9,10]}$, 即

$$
\frac{\lambda_{v}^{2}}{\lambda_{g} \lambda_{l}}=\frac{\lambda_{p} \lambda_{l}}{\lambda_{v}}=1 .
$$

本试验取 $\lambda_{l}=1$, 而 $\lambda_{g}=1, \lambda_{\rho}=2.64$, 则由式(2)可知, 当 $\lambda_{v}=1$ 即模型和原型渗流液体的黏度比尺为 1 时，在 初始条件和边界条件相似的情况下，即可满足模型 与原型的流动相似.

模拟液: 由于渗流过程中铝熔体温度变化范围为 $660 \sim 700{ }^{\circ} \mathrm{C}$, 相应的黏度变化范围为 $2.9 \times 10^{-3} \sim 2.7 \times$ $10^{-3} \mathrm{~Pa} \cdot \mathrm{s}, \Delta v<0.2 \times 10^{-3} \mathrm{~Pa} \cdot \mathrm{s}^{[14,15]}$, 而且渗流过程所需 
时间非常短 $(<3 \mathrm{~s})$, 换热过程不能充分进行, 所以可 将铝液的黏度值简化为常量来处理. 本文选取铝液 的黏度为 $v_{700}=2.7 \times 10^{-3} \mathrm{~Pa} \cdot \mathrm{s}$. 试验选取 $20^{\circ} \mathrm{C}$ 的水作 为模拟液体, 则

$$
\lambda_{v}=\frac{v_{\mathrm{Al}}(700)}{v_{\text {水 }}(20)}=\frac{2.7 \times 10^{-3} / 2.64 \times 10^{3}}{1.003 \times 10^{-6}}=1 .
$$

\section{2 渗流模拟试验结果及与熔体渗流结果比较}

\subsection{1 渗流模拟试验结果}

通过观察和摄像得知，在所有的试验中，渗流液 面均为水平面, 与型腔壁面相垂直, 且沿铅垂方向推 进. 铝熔体渗流过程的模拟试验结果列于表 3 .

\section{据此可得}

渗流液面推进的位移 $L$ 与时间 $t$ 的关系

$$
L=1.045 t^{\frac{1}{4}}-1.010 \text {; 通式为 } L=a_{1} t^{\frac{1}{4}}+b_{1} \text {. }
$$

渗流液面推进速度 $u$ 与时间 $t$ 高度 $I$ 的关系

$$
u=0.668 t^{-\frac{3}{4}} \text {; 通式为 } u=a_{2} t^{-\frac{3}{4}} \text {. }
$$

联立解式 (3) 和 (4) 得

$$
\begin{aligned}
L= & 0.668 u^{-\frac{1}{3}}-1.010 \text { 或 } u=0.299(L+1.010)^{-3} . \\
& \text { 通式为 } L=a_{3} u^{-\frac{1}{3}}+b_{3} \text { 或 } u=a_{4}\left(L+b_{4}\right)^{-3} .
\end{aligned}
$$

渗流压强 $p$ 与时间 $t$ 的关系如表 4 所示.

以充满型腔时为例, 传感器 (5) 压强变化曲线(数 据见表 4)可表示为

$$
\rho=0.574 t^{\frac{1}{4}}-0.555 ; \text { 通式为 } \rho=a_{5} t^{\frac{1}{4}}+b_{5} .
$$

渗流压强 $p$ 与渗流高度 $L$ 的关系, 可联立解式 (4)(6)得

$$
p=0.574 L-0.555 ; \text { 通式为 } p=a_{6} L+b_{6} \text {. }
$$

式(3) (7)表明渗流速度、压强随时间、空间的变化. 式 中的常数项随外加渗流气压而变化.

\subsection{2 熔体在多孔介质中的渗流过程与模拟渗流 试验结果对比}

根据流体流动相似原理，由模拟试验得到原型 中铝液的渗流现象和规律, 并在原型得到验证, 充满 $150 \mathrm{~mm}$ 填料颗粒层的模拟渗流及原型渗流时间，一 组为 $1.58,1.60 \mathrm{~s}$; 另一组为 $1.52,1.56 \mathrm{~s}$, 二者基本相 同，由于忽略了铝熔体的温度及黏度变化，原型渗流 时间略长. 证明了时间比尺 $\lambda_{\mathrm{t}}=1$ 和速度比尺 $\lambda_{\mathrm{u}}=1$.

坩埚内铝液表面压强值可以直接测得，约为水 箱气液表面压强值 $p_{0}$ 的 2.64 倍，当充满颗粒层高 150

\begin{tabular}{|c|c|c|c|c|c|c|}
\hline$t / \mathrm{s}$ & 0.88 & 1.17 & 1.52 & 1.94 & 2.46 & 备注 \\
\hline \multirow{5}{*}{$p / \mathrm{kPa}$} & 0 & 4.153 & 8.307 & 12.257 & 16.512 & 传感器(5) \\
\hline & & 0 & 4.66 & 8.104 & 12.359 & 传感器(4) \\
\hline & & & 0 & 4.559 & 7.598 & 传感器(3) \\
\hline & & & & 0 & 4.052 & 传感器(2) \\
\hline & & & & & 0 & 传感器(1) \\
\hline
\end{tabular}
$\mathrm{mm}$ 时，模拟及原型渗流压强，一组为 $9.32 、 25.02 \mathrm{kPa}$, 压强比尺为 2.69 ; 另一组为 $9.83 、 26.64 \mathrm{kPa}$, 压强比 尺为 2.71 , 表明压强比尺接近 $\lambda_{p}=2.64$.

\section{表 3 模拟渗流试验数据}

\begin{tabular}{ccccccc}
\hline$t / \mathrm{s}$ & 0.88 & 1.17 & 1.52 & 1.94 & 2.46 & 备注 \\
\hline$L / \mathrm{m}$ & 0.005 & 0.075 & 0.150 & 0.225 & 0.300 & \\
$\nu / \mathrm{m} \cdot \mathrm{s}^{-1}$ & 0.288 & 0.232 & 0.191 & 0.159 & 0.133 & 16.512 \\
$p / \mathrm{kPa}$ & 0 & 4.153 & 8.307 & 12.257 & 62.40 & 传感器 5 \\
$R \mathrm{e}$ & 135.12 & 108.84 & 89.61 & 74.60 & 计算数据 \\
\hline
\end{tabular}

表 4 模拟渗流压强 $p$ 与时间 $t$ 的关系 


\subsection{3 熔体在多孔介质中渗流模拟的新进展}

采用模拟试验, 在相似原理的指导下用常温模 拟液代替高温铝液, 研究颗粒直径对相似准则的影 响, 在 $\lambda_{\mathrm{L}}=1$ 和 0.5 时, 采用模拟试验和熔体试验验证, 系统研究了铝液在 $0.5 \sim 3.5 \mathrm{~mm}$ 颗粒中的渗流规律. 得到铝液渗流过程的相似准则为: 对直径 $1.5 \sim 3.5 \mathrm{~mm}$ 的颗粒介质为Froude 准则, 对直径 $0.5 \mathrm{~mm}$ 的颗粒介 质为 Reynolds准则 ${ }^{[9]}$. 对于较大的颗粒介质应采用较 小的渗流驱动压力，以避免颗粒被熔体全部包裹，而 不能形成多孔铝合金 ${ }^{[14]}$.

上述在多孔介质中的渗流过程模拟都是在宏观 大尺度范围，以达西定律为基础，进一步发展了小尺 度有限颗粒范围二维微管的渗流过程物理模拟 ${ }^{[15]}$ 及 渗流过程数值模拟新方法 ${ }^{[8]}$, 获得更为精确的结果.

综上所述，根据相似原理，采用合适的准则，揭 示了铝熔体渗流的规律，与铝熔体渗流试验相吻合， 指导了多孔铝合金的制备.

\section{4 多孔铝合金孔结构物理模型理论及试验 结果}

\section{1 多孔铝合金总孔隙率}

总孔隙率 $P r$ 分为理论计算孔隙率 $P r_{\mathrm{c}}$ 及试验孔隙 率 $P r_{\mathrm{e}}$ :

$$
P r_{\mathrm{c}}=P r_{\text {堆积 }}+P r_{\text {附加 }}+P r_{\text {凝固 }}
$$

多孔铝合金孔径由可去除的颗粒孔径d控制，但 另一重要参数总孔隙率 $P r_{\mathrm{e}}$, 传统认为等于颗粒堆积

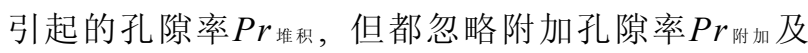
$P r_{\text {㠜固 }}\left(\right.$ 约占 $5 \%$ ), 其中 $P r_{\text {堆积 }}\left(\right.$ 约占总孔隙率 $90 \%$ )及 $P r_{\text {漩 }}$

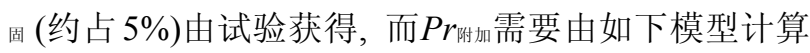
获得.

\section{2 球形孔多孔铝合金附加孔隙率的理论模型 [4]}

建立多孔铝合金孔结构的模型，本质上是从球 形孔入手建立附加孔隙率的理论，只有当颗粒不被 铝合金熔体完全包围凝固以形成通孔度，才可能去 除填料颗粒，形成通孔多孔铝合金，通孔度形成的附 加孔隙率称之为 $P r$ 附加, 它数值甚少, 约占 $5 \%$, 但是 形成通孔的关键, 它与渗流驱动力 $\Delta P$, 熔体与颗粒 的润湿情况及颗粒直径密切相关.
假设形成球形孔颗粒 $(2 R=1.3 \sim 5.5 \mathrm{~mm})$ 为刚性球, 熔体在液态渗流过程颗粒保持球形, 温度不变, 并且 熔体以平面形式渗流.

熔体在两相切刚性球间简化剖面模型见图 4, 5 .

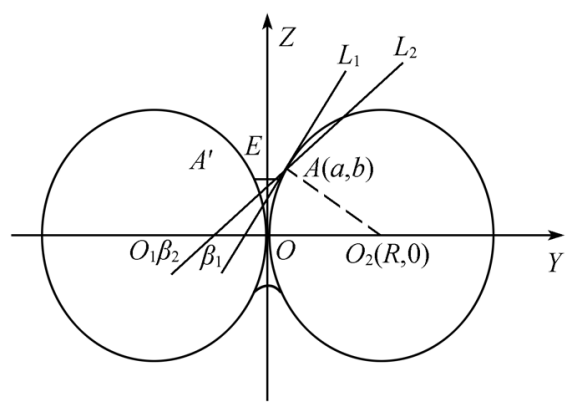

图 4 两刚性球交界处液-气界面的 $Y-Z$ 截面

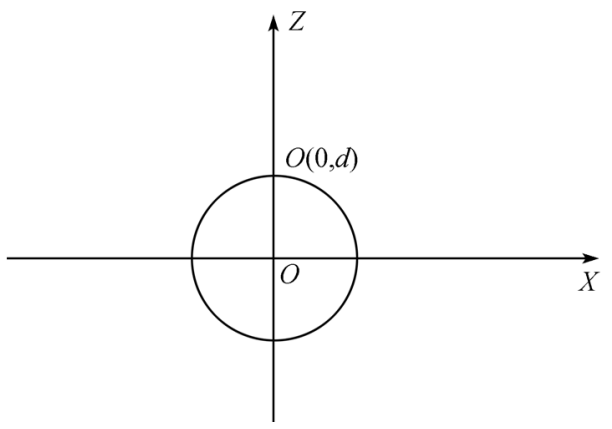

图 5 两刚性球交界处的液-气界面 $X-Z$ 截面

以切点 $O$ 为坐标原点, 两球的球心 $O_{1}$ 和 $O_{2}$ 的连 线为 $Y$ 轴, 过 $O$ 点做 $Z$ 轴垂直于 $Y$ 轴, $X$ 轴为过 $O$ 点 垂直于纸面. 图中点 $A$ 和 $A^{\prime}$ 为铝合金熔体液面与圆 $O_{1}$ 和 $O_{2}$ 的交点, 点 $E$ 为铝合金熔体液面与 $Z$ 轴的交 点. 过点 $A$ 作圆 $O_{2}$ 的切线 $L_{1}$ 和铝合金熔体液面 $A^{\prime} E A$ 的切线 $L_{2} . L_{1}$ 和 $L_{2}$ 与 $Y$ 轴的夹角分别为 $\beta_{1}$ 和 $\beta_{2}, L_{1}$ 和 $L_{2}$ 之间的夹角为 $\beta$. 设铝合金熔体与填料粒子之间的 润湿角为 $\theta$, 则 $\theta=180^{\circ}-\beta$, 且 $\beta=\beta_{1}-\beta_{2}$. 设弯曲 液面 $A^{\prime} E A$ 上下的压力差为 $\Delta P$, 对曲线上的 $E$ 点应用 LaPlace 方程, 有

$$
\Delta P=\sigma\left(1 / r_{1}+1 / r_{2}\right)
$$

式中 $\sigma$ 为铝合金熔体的表面张力, $r_{1}$ 为弯曲液面 $A^{\prime} E A$ 在 $E$ 点的曲率半径, $r_{2}$ 为 $E$ 点绕 $Y$ 轴的回转半径, 即通 孔半径 $d$, 如图 4 所示. 
由图 4 可知, 弯曲液面 $A^{\prime} E A$ 在 $Y-Z$ 截面内的截 线不可能为圆, 从 $E$ 点到 $A$ 点, 垂直于 $Y-Z$ 截面的曲 率圆的曲率半径不断增大, 则 $Y-Z$ 截面的曲率圆的曲 率半径也不断增大, 现做简化处理, 设截线为抛物线, 方程式为

$$
Z=K Y^{2}+d,
$$

式中 $k, d$ 为常数.

球形孔附加孔隙率计算公式:

$$
P r_{\text {附加 }}=P r_{\text {堆积 }} n V_{a} / V_{q} \text {. }
$$

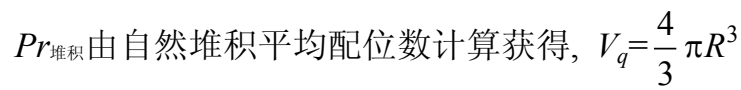
为填料粒子的体积, $V_{a}$ 为附加孔隙的体积 ${ }^{[3]}$.

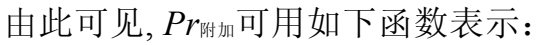

$$
P r_{\text {附加 }}=f\left(k, a, b, d, \Delta p, \theta, \sigma, R, P r_{\text {堆积 }}, n\right),
$$

其中 $k, a, b, d$ 和 $n$ 由模型参数经过数值计算获得; $\Delta p, \theta, \sigma$ 和 $R$ 由工艺参数和物性参数获得, $P r_{\text {堆积由试验 }}$ 获得. 将上述参数和数据代入(11)式, 计算得出 $P r$ 附加.

由表 5 可见, 球形孔多孔铝合金 $P r_{\mathrm{c}}$ 与 $P r_{\mathrm{e}}$ 吻合良 好，误差不大于 $5.66 \%$; 对于多角形多孔铝合金引入 形状系数获得多角形物理模型, 同样获得了 $P r_{\mathrm{c}}$ 与 $P r_{\mathrm{e}}$ 良好吻合的结果.

综上所述，建立多孔铝合金孔隙率的物理模型， 揭示了附加孔隙率与渗流驱动力的关系, 并与试验
结果吻合，指导多孔铝合金的孔隙率控制.

\section{5 梯度孔径及周期调制通孔多孔铝合金 5$]$}

由毫米级颗粒形成的多孔铝合金的孔结构，在 毫米级孔腔之间有尺寸更小的通孔度. 当声波进入 毫米级孔腔后再进入更小的通孔度，空气膨胀及压 缩而衰减声波能量，因而可以将通孔多孔铝合金看 作吸收体组合, 具有较好的空气吸声性能.

但单一孔径多孔铝合金在吸声方面具有频率选 择性, 吸声系数在宽频率范围呈现周期性波动. 依据 多孔材料的声波阻抗特性，具有渐变阻抗或周期阻 抗的多孔材料具有更佳的宽频吸声效果.

文献[16]提出了单一孔结构多孔铝的吸声模型, 根据该模型，可以获得其吸声系数表达式:

$$
\alpha=\frac{4 R_{n} / \rho_{0} c_{0}}{\left(1+R_{n} / \rho_{0} c_{0}\right)^{2}+\left(M_{n} / \rho_{0} c_{0}\right)^{2}},
$$

其中 $\rho_{0}$ 为空气的密度, $c_{0}$ 为空气中的声速, $R_{n}$ 为 $n$ 个孔 的声阻率, $M_{n}$ 为 $n$ 个孔的声抗. 式(13)启发了梯度孔径 及周期调制多孔铝合金在声学领域的发展.

\section{1 梯度孔径调制多孔铝合金}

\subsection{1 梯度调制多孔铝合金的制备原理及孔结构

\begin{tabular}{|c|c|c|c|c|c|c|c|}
\hline $2 R / \mathrm{mm}$ & $p / \mathrm{kPa}$ & $\operatorname{Pr}_{e} / \%$ & $P r_{\text {堆积 }} / \%$ & $P r_{\text {附加 }} / \%$ & $P r_{\text {凝固 }} / \%$ & $P r_{\mathrm{C}} / \%$ & $\left(\left|P r_{\mathrm{C}}-P r_{e}\right| / P r_{\mathrm{C}}\right) / \%$ \\
\hline $1.6 \sim 0.9$ & 7.89 & 75.1 & 68.9 & 3.3 & 3.1 & 75.3 & 0.27 \\
\hline $1.6 \sim 0.9$ & 8.55 & 72.8 & 69.9 & 3.1 & 3.0 & 76.0 & 4.21 \\
\hline $2.6 \sim 1.6$ & 5.26 & 72.0 & 57.0 & 2.9 & 3.4 & 73.3 & 0.95 \\
\hline $2.6 \sim 1.6$ & 7.89 & 68.1 & 66.8 & 1.8 & 3.5 & 72.2 & 5.66 \\
\hline $3.8 \sim 3.0$ & 2.63 & 74.3 & 66.2 & 3.5 & 3.4 & 73.1 & 1.64 \\
\hline $3.8 \sim 3.0$ & 5.26 & 67.2 & 65.6 & 1.7 & 3.7 & 70.9 & 5.22 \\
\hline $5.8 \sim 3.8$ & 1.97 & 72.1 & 67.4 & 3.4 & 3.3 & 74.1 & 2.70 \\
\hline $5.8 \sim 3.8$ & 3.94 & 69.0 & 67.4 & 1.6 & 3.5 & 72.5 & 4.83 \\
\hline
\end{tabular} 参数}

控制堆积颗粒直径 $d$ 沿渗流长度 $x$ 方向，呈梯度

表 5 球形孔多孔铝合金实测孔隙率 $P r_{e}$ 与理论孔隙率 $P r_{\mathrm{C}}$ 的比较

表 6 梯度孔径多孔铝合金试样的结构参数

\begin{tabular}{cccc}
\hline 试样 & 孔隙率 $/ \%$ & 孔径范围 $/ \mathrm{mm}$ & 梯度孔径曲线 \\
\hline $\mathrm{a}$ & 67.12 & $1.25 \sim 0.5$ & $D=0.00046875(x-40)^{2}+0.5$ \\
$\mathrm{~b}$ & 66.83 & $1.25 \sim 0.5$ & $D=0.001875(x-20)^{2}+0.5$ \\
$\mathrm{c}$ & 66.36 & $1.25 \sim 0.5$ & $D=0.0075(x-10)^{2}+0.5$ \\
\hline
\end{tabular}


变化, $d=f(x)$, 按照前述多孔铝合金的制备方法，即 可获得孔径按照 $d=f(x)$ 变化的梯度孔径多孔铝合金.

梯度孔径多孔铝合金的孔结构如图 6,7 所示.

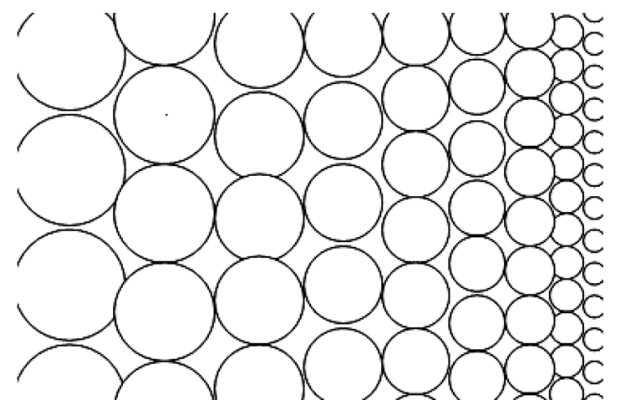

图 6 孔径梯度调制多孔铝合金模型

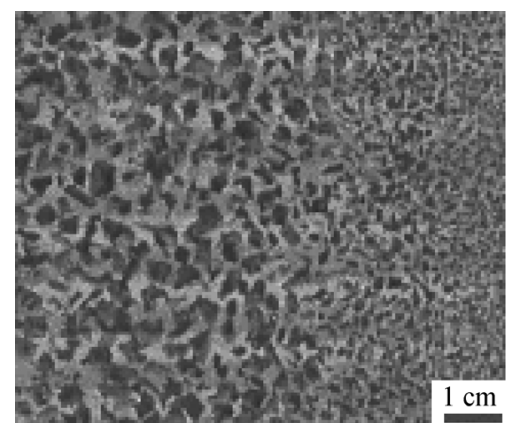

图 7 梯度孔径多孔铝合金试样剖面图

\subsection{2 吸声系数的试验值与理论值对比}

采用驻波管法，按照标准 GBJ88-1985《驻波管 吸声系数与声阻抗率测量规范》测量多孔铝的空气声 吸收系数. 测量时, 声波从大孔端面进入多孔铝合金. 其试验值与理论值如图 8 所示, 其中孔结构参数如表 6 所示.

由图 8 可见, 各试样空气吸声系数的试验值与理 论值基本吻合，表明能够利用理论模型对梯度孔径 多孔铝合金的吸声性能进行表征. 梯度孔径多孔铝 合金具有预期的宽频吸声效果，其空气吸声系数与 样品的梯度及厚度有密切关系。

\section{2 孔径周期调制多孔铝合金}

\subsection{1 周期调制多孔铝合金的制备原理及孔结构}

控制填料颗粒直径 $d_{1} 、 d_{2}$ 在渗流方向呈周期变化, 按照前述多孔铝合金的制备方法即可获得孔径呈周

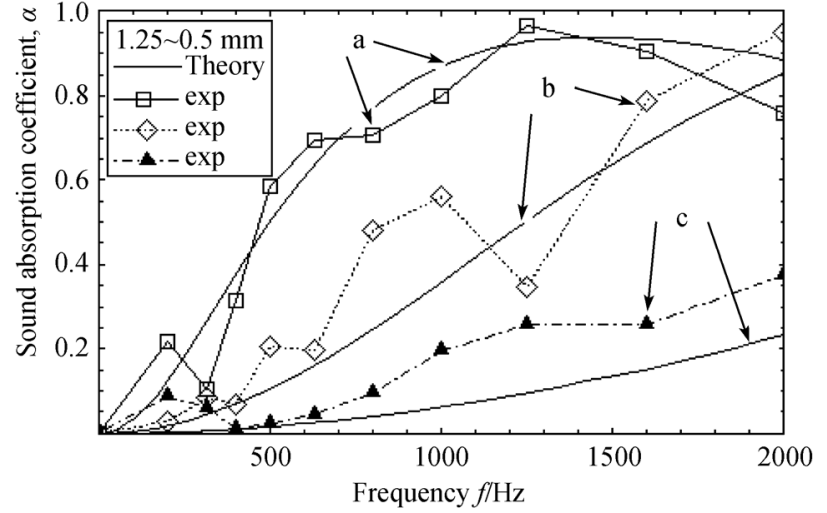

图 8 梯度孔径多孔铝空气吸声系数的试验值与理论值 对比

期调制的多孔铝合金.

1 个调制周期单元包括 1 个小孔径层(孔径为 $d_{S}$, 层厚为 $\delta_{d S}$ ) 和 1 个大孔径层 (孔径为 $d_{L}$, 层厚为 $\delta_{d L}$ ), 如 图 9,10 所示. 计算表明, 在试验采用的渗流制备参 数下，由于粒径不同造成的附加孔隙率的差别在 $1 \%$ 以下，因而在吸声模型的计算中可以将大、小孔径

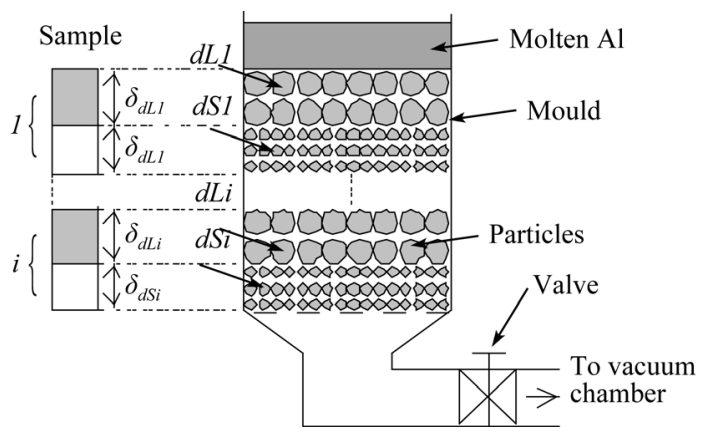

图 9 孔径周期调制多孔铝合金试样

试样图中的灰色与白色部分分别对应大孔径分层和小孔径分层

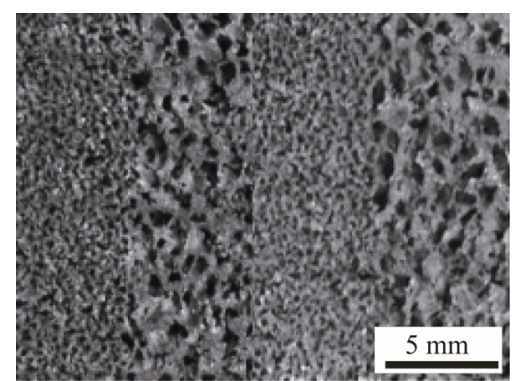

图 10 孔径周期调制多孔铝试样的剖面图 
表 7 孔径周期调制多孔铝样品的结构参数

\begin{tabular}{|c|c|c|c|c|c|c|c|}
\hline 样品 & 孔隙率/\% & 孔径 $/ \mathrm{mm}$ & \multicolumn{4}{|c|}{ 孔径层厚度 $/ \mathrm{mm}^{\mathrm{a})}$} & 样品厚度/mm \\
\hline $\mathrm{a}$ & 65.28 & $1.25,0.5$ & 10 & & 10 & & 20 \\
\hline $\mathrm{b}$ & 65.28 & $1.25,0.5$ & 10 & & 10 & & 20 \\
\hline $\mathrm{c}$ & 66.01 & $1.25,0.5$ & 5 & 5 & 5 & 5 & 20 \\
\hline d & 66.01 & $1.25,0.5$ & 5 & 5 & 5 & 5 & 20 \\
\hline
\end{tabular}

a) 表中的灰色与白色背景分别对应大孔径层和小孔径层

层的孔隙率用相同参数进行表征.

吸声系数的测量采用驻波管法按照标准 GBJ881985, 样品参数如表 7 所示.

\subsection{2 吸声系数的实验值与理论值比较}

周期调制多孔铝合金空气吸声系数的试验值与 理论计算值的对比如图 11 所示.

由图 11 可见, 各试样吸声系数的试验值与理论 值基本吻合，表明能够利用理论模型对孔结构周期
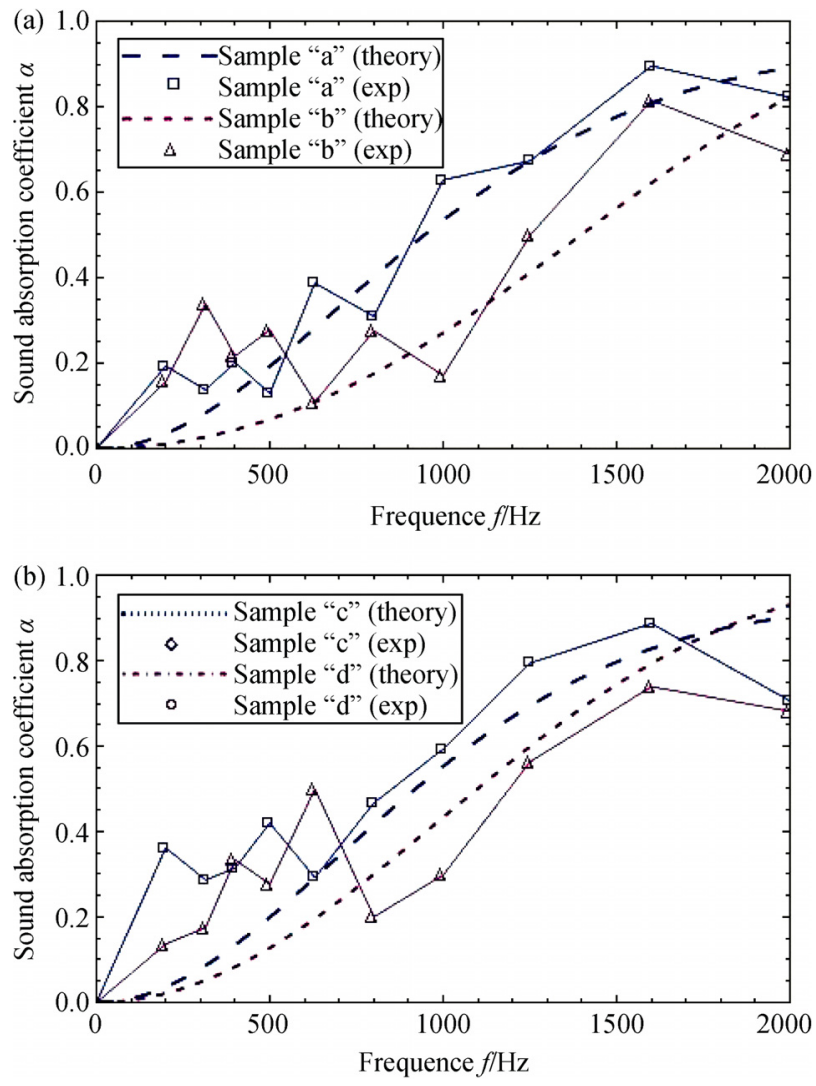

图 11 试验值与理论值的比较

(a) 试样 “a” “b”; (b) 试样 “c” “d”
调制多孔铝的吸声性能进行表征.

周期调制可提高多孔铝合金的吸声性能; 随着 小孔径层与大孔径层厚度比的增大, 吸声性能逐渐 提高; 在试样厚度相同时, 通过减小每层的厚度来增 加调制周期，能够使吸声曲线整体向低频方向略微 偏移. 在厚度及孔径范围相同时，梯度及周期调制多 孔铝合金的吸声性能在宽频率范围优于单一孔径多 孔铝合金，可解决单一孔径多孔铝合金空气吸声系 数随频率波动的问题.

综上所述，在单一孔径多孔铝合金的基础上发 展了梯度孔径及周期调制多孔铝合金，其吸声系数 理论值与试验值基本吻合; 吸声性能均优于单一孔 径多孔铝合金.

\section{6 结论}

（1）铝合金熔体在可除去多孔介质中渗流制备 某种高技术多孔铝合金，主要控制熔体温度和颗粒 预热温度，见式(1), 控制熔体渗流压力 $\Delta P$ 以使熔体 不完全包围颗粒，以形成通孔度，这是形成多孔铝合 金的关键.

（2）根据相似原理，见式(2)，采用合适的模型模 拟液及装置，揭示模拟液在多孔介质颗粒间渗流的 规律，见式(3) (7), 两者结果吻合良好, 并发展了物 理模拟及数值模拟新方法，从而为多孔铝合金的制 备奠定了基础.

(3) 在多孔铝合金孔隙率组成式(8)的基础上，建 立了附加孔隙率的物理模型及表达式，见式(11) (12), 获得了理论与试验值良好吻合的结果，见表 5.

(4) 以单一孔径多孔铝合金为基础发展了梯度 孔径及周期调制两类新型多孔铝合金，见图 6, 9, 声 吸收试验结果与理论计算吻合良好，见图 8,11, 两者 都提高了声吸收性能，后者制备更为简单，从而展示 了应用前景. 
致谢本工作得到国家重点基础研究发展计划项目(批准号：2006CB601201)、国家自然科学重点基金(批准 号: 50231010, 19472020)资助.

\section{参考文献}

1 Kucheck H A. US Patent 3236706, 1996

2 何德坪，王永进，陈策，杨东辉。多孔铝合金及制备方法. 中国, CN 1161486C. 2004

3 闻德蒜, 张勇, 何德坪, 舒光冀, 陶同康. 金属基复合材料及多孔金属的渗流制备模拟法. 中国, CN 1041847C. 1993

4 杨东辉, 何德坪. 多孔铝合金的孔隙率. 中国科学 B 辑: 化学, 2001, 31(31): 265-271

5 何德坪，黄可，刘伟伟，何思渊，王联风，顾菲菲。孔结构调制通孔多孔铝及铝合金和其制备方法. 中国, CN101104894. 2008

6 何德坪，陈锋。特殊预制块法制备通孔泡沫铝合金.中国, CN1019027B. 1993

7 何德坪, 张伟开, 何思渊, 黄可, 刘伟伟, 卢天健. 利用塑料颗粒制造高孔隙率通孔多孔铝合金的方法及装置. 中国, CN1876861. 2006

8 张伟开. 高孔隙率多孔铝合金的形成及其性能研究. 南京: 东南大学, 2006

9 程桂萍. 多孔金属的制备及液态金属渗流动力学研究. 南京: 东南大学, 1997

10 张勇. 多孔铝的渗流铸造、模拟实验及水下声吸收特性. 南京: 东南大学, 1993

11 Mortensen A, Masurl L J. Infiltration of fibrous preforms by a pure metal. Part I , Theory. Metall Trans, 1989, 20A(11): 2535[DOI]

12 Masur L J, Mortensen A, Cornie J A, Flemings M C. Infiltration of fibrous preforms by a pure metal: Part II . Experiment. Metall Trans A, 1989, 20A: 2549-2557

13 何德坪, 闻德䔉, 张勇, 舒光冀. 铝熔体在多孔介质中的渗流过程. 材料研究学报, 1997, 11(2): 113-119

14 闻德蒜. 工程流体力学(上册). 北京: 高等教育出版社, 1993

15 刘兵, 何思渊, 何德坪. 铝合金熔体在多孔介质中的二维微管渗流模型. 材料研究学报, 2006, 20(4): 389-393

16 Lu T J, Chen F, He D P. Sound absorption of cellular metals with semiopen cells. J Acoust Soc Am, 2000, 108: 1697-1709 [DOI] 